Voix et Images

volxetimages

\title{
Bibliographie de Michel Marc Bouchard
}

\section{Sébastien Roy}

Volume 33, numéro 1 (97), automne 2007

Michel Marc Bouchard

URI : https://id.erudit.org/iderudit/017531ar

DOI : https://doi.org/10.7202/017531ar

Aller au sommaire du numéro

Éditeur(s)

Université du Québec à Montréal

ISSN

0318-9201 (imprimé)

1705-933X (numérique)

Découvrir la revue

Citer ce document

Roy, S. (2007). Bibliographie de Michel Marc Bouchard. Voix et Images, 33(1),

97-112. https://doi.org/10.7202/017531ar d'utilisation que vous pouvez consulter en ligne.

https://apropos.erudit.org/fr/usagers/politique-dutilisation/ 


\title{
B I BLIOGR A PHIE DE MICHEL MARC BOUCHARD
}

\author{
$+++$ \\ SÉBASTIEN ROY \\ Université du Québec à Montréal
}

\section{I . E U V R E S}

\section{I . 1.1. Th é ât re}

+ La contre-nature de Chrysippe Tanguay, écologiste, préf. d'Yves Dubé, Montréal, Leméac, coll. "Théâtre/Leméac», 1984, 70 p.

+ La poupée de Pélopia, préf. d’Yves Dubé, Montréal, Leméac, coll. «Thêâtre/Leméac», 1985, $83 \mathrm{p}$.

+ Rock pour un faux-bourdon, préf. de Claude Poissant, Montréal, Leméac, coll. «Théâtre/ Leméac», 1987, 127 p.

+ Les feluettes ou La répétition d'un drame romantique, préf. de l'auteur intitulée «Réflexion sur les bords d'une mer intérieure», Montréal, Leméac, coll. «Thêâtre/Leméac», 1987, 125 p.

+ Les muses orphelines, préf. de l'auteur intitulée «Ma galerie de tableaux», Montréal, Leméac, coll. «Théâtre», 1989, 118 p.

+ Soirée bénéfice pour ceux qui ne seront pas là en l'an 2000, Ottawa, Centre national des arts, 1990.

+ Les grandes chaleurs, Montréal, Leméac, 1993, 97 p.

+ BOUCHARD, Michel Marc, et Noëlle RENAUDE, Les muses orphelines, DALPÉ, Jean-Marc, et Eugène DURIF, Le chien, préf. de Lorraine Hébert, Montreuil-sous-Bois [France], Éditions thêâtrales, coll. "Répertoire Contemporain», 1994, 120 p.

+ Le désir, Belœil [Québec], Thêâtre la Fenière; L'Ancienne-Lorette [Québec], Théâtre des Hirondelles, 1995.

+ Les muses orphelines, nouv. version, Montréal, Leméac, coll. «Théâtre», 1995, 81 p.

+ Le voyage du couronnement, Montréal, Leméac, coll. "Thêâtre», 1995, 118 p.

+ Une entreprise amoureuse, Montréal, L'Ancienne-Lorette [Québec], Thêâtre des Hirondelles, 1996.

+ Pierre et Marie... et le démon, Rougemont [Québec], Théâtre de Rougemont, 1997.

+ Le chemin des passes dangereuses, Montreuil-sous-Bois [France], Éditions thêâtrales, coll. «Répertoire Contemporain», 1998, 64 p.

+ Le chemin des Passes-dangereuses: tragédie routière, préf. de l'auteur intitulée «Pourquoi écrire Le chemin des Passes-dangereuses », Montréal, Leméac, coll. «Théâtre», 1998, 71 p.

+ Les aboyeurs, Saint-Nicolas [Québec], Théâtre Beaumont-St-Michel, 1999.

+ Les papillons de nuit: comédie, Montréal, Leméac, coll. «Théâtre», 1999, 109 p. 
+ Sous le regard des mouches, préf. de l'auteur intitulée «Le jeu de la mort», Montréal, Leméac, coll. «Théâtre», 2000, 93 p.

+ Sous le regard des mouches/Le voyage du couronnement, Montreuil-sous-Bois [France], Éditions théâtrales, coll. «Répertoire Contemporain», 2001, 160 p.

+ Les manuscrits du déluge, préf. de l'auteur intitulée "Déluge et mémoire», Montréal, Leméac, coll. "Théâtre/Leméac», 2003, 83 p.

+ Les muses orphelines, version pour la France de Noëlle Renaude, Montreuil-sous-Bois [France], Éditions théâtrales, coll. «En scène», 2006, 64 p.

+ Le peintre des madones ou La naissance d'un tableau, Montréal, Leméac, coll. «Théâtre/ Leméac ", 2004, 99 p.

+ Les porteurs d'eau, Montréal, Leméac, coll. "Théâtre/Leméac», 2004, 83 p.

+ Les manuscrits du déluge, Montreuil-sous-Bois [France], Éditions thêâtrales, coll. "En scène», 2006, 64 p.

+ Des yeux de verre, Montréal, Leméac, coll. "Théâtre/Leméac», 2007, 66 p.

+ La nouvelle fabuleuse ou Les aventures d'un Flo, Ville Saguenay [Québec], Théâtre du Palais municipal, 2007.

\section{1.2. Théâtre jeunesse}

+ L'histoire de l'oie, préf. de l'auteur intitulées «Histoire pour enfants humiliés» ainsi que «L'aventure», Montréal, Leméac, coll. «Théâtre jeunesse», 1991, 51 p.

+ L'histoire de l'oie, Montréal, inédit, photocopies, 1991, 44 p. [extrait annoté pour les pages 1 à 27, disponible au CEAD]

+ Histoire de l'oie, Montreuil-sous-Bois [France], Éditions théâtrales, coll. «Théâtrales jeunesse», 2001, $64 \mathrm{p}$

\section{1.3. En revue}

+ . Les limbes», Les Cahiers de Prospero, n 6, 1995, p. 97.

+ «Le jade et l'ébène», Mœbius. Contes urbains, n 75, 1997 (hiver), p. 21-25.

\section{2. EUURES EN COLLABORATION}

\section{2.1. Th é ât re}

+ « Du haut de ses vingt ans», 20 ans, Montréal, CEAD et VLB éditeur, 1985.

+ La visite ou Surtout, sentez-vous pas obligés de venir!, avec Robert BELLEFEUILLE, rev. et corr. en 1993 par Benoît Lagrandeur, Sudbury [ON], Thêâtre du Nouvel-Ontario et Théâtre de la Vieille 17, Ottawa, 1987.

+ "L'inachevé - exercice sur l'orgueil», Les huit péchés capitaux (éloges), en collaboration avec Normand CANAC-MARQUIS, Jean-François CARON, René Richard CYR, René-Daniel DUBOIS, Larry TREMBLAY, Michel TREMBLAY et Lise VAILLANCOURT, Montréal, Dramaturges éditeurs, 1997, p. 105-112.

\section{2.2. Livre d'art}

+ BOUCHARD, Michel Marc, et Claire BEAULIEU, «Le héros», dans Coin du banc, sous la dir. de Jacques Fournier, Montréal, Éditions Roselin, 1996, vol. 5 de 8. 


\section{I.2.3. Expositions}

+ Chaîne culturelle FM de Radio-Canada à Québec et Aquarium du Québec, Ludovica : histoires de Québec, scénario de Michel Marc Bouchard, Québec, Musée de l'Amérique française. 5 mai au 4 septembre 2000.

+ CHAMBERLAND, Line, et al., Mythes et réalités des cultures gaies et lesbiennes: exposition historique et culturelle, scénario de Michel Marc Bouchard et Nicole Brossard, Montréal, Maison de la culture Marie-Uguay, 1997, 62 p. Exposition du 11 septembre au 26 octobre 1997, Maison de la culture Marie-Uguay.

+ CREVIER, Isabelle, et Sophie MONTREUIL (dir.), Tous ces livres sont à toi! : de l'œuvre des bons livres à la Grande Bibliothèque (1844-2005), Montréal, Bibliothèque nationale du Québec;

Québec, Presses de l'Université Laval, 2005, 181 p. Ouvrage de l'exposition du même nom avec Nicole Lemay pour commissaire et Michel Marc Bouchard comme directeur artistique.

+ LAFORGE, Valérie, Talons et tentations, Québec, Musée de la civilisation et Éditions Fides, coll. «Images de sociétés», 2001, 128 p. Exposition de novembre 2001 à septembre 2002, direction artistique de Michel Marc Bouchard.

\section{2.4. Films et enregistrements vidéo}

+ Les muses orphelines, mise en scène par René Richard Cyr, Montréal, Théâtre d'Aujourd'hui [Fonds Thêâtre d'Aujourd'hui], 1988, DVD, 1 h 42 min 11 s.

+ L'histoire de l'oie, mise en scène par Daniel Meilleur, Montréal, Production Les Deux Mondes, 1992, VHS, 56 min $30 \mathrm{~s}$, copie DVD.

+ BOURBONNAIS, Jean, et Marie CADIEUX, Paroles en jeu: hommage à la dramaturgie francoontarienne, Toronto, Médiatique inc., 1998, 52 min, VHS, son, coul. [Participation de M. M. Bouchard à la narration.]

+ FAVREAU, Robert, Les muses orphelines, Montréal, Film Tonic; Westmount [Québec], Christal Films, 2000, 107 min.

+ GREYSON, John, Lilies - Les feluettes, Canada, Tryptich Media, 1996, 95 min, 35 mm, son, coul.

\section{I.2.5. En registrements sonores}

+ Les muses orphelines, réal. de Line Meloche et de René Richard Cyr, mus. de Michel Smith, Montréal, Audiogram, 1997, 2 disques, 1 h 29 min 38 s.

+ Le peintre des madones ou La naissance d'un tableau, Montréal, La Magnétothèque, 2006, 1 disque, 2 h 7 min.

+ Les huits [sic] péchés capitaux: éloges, Montréal, La Magnétothèque, 2007, 1 disque.

\section{3. A U T RES}

\section{3.1. Let tre}

+ Lettre d'un jeune adulte à ses parents [présentée exclusivement pour la Journée nationale de lutte contre l'homophobie], Montréal, Fondation Émergence, 2005, 2 p., Adresse URL: http://www.homophobie.org/utilisateur/documents/homophobie/10minutes/lettre_mmb.pdf [consultée en ligne le $1^{\mathrm{er}}$ juin 2007].

\section{3.2. Com mentaires}

+ «La critique journalistique en région entre le commentaire et le superlatif», Jeu, n 40, 1986, p. 47.

+ «Écrire», Théâtre Québec, 1995 (décembre), p. 12. 


\section{I.3.3. Matériel pédagogique}

+ L'écriture dramatique, l'écriture scénique, Ottawa, Thêâtre-Action, 1989, 69 p.

\section{I.3.4. Présence dans des ouvrages de référence}

+ Répertoire des auteurs dramatiques. Dramaturgie québécoise et franco-ontarienne disponible au CEAD, Montréal, Centre des auteurs dramatiques (CEAD), Adresse URL: http://www.cead.qc.ca/Repertoire_Auteurs.html [Mise à jour datant du 25 mai 2007].

+ Centre des auteurs dramatiques, Théâtre québécois, Montréal, Centre des auteurs dramatiques, 1990-1994.

+ BERNANOCE, Marie, À la découverte de cent et une pièces, préf. de Jean-Claude Lallias, Montreuil-sous-Bois, Éditions théâtrales; Grenoble, SCÉRÉN-CRDP de l'académie de Grenoble, 2006, 540 p.

+ CHARTIER, Daniel, Guide de culture et de littérature québécoises, Québec, Éditions Nota bene, 1999, $344 \mathrm{p}$.

+ CHARTIER, Daniel, Le guide de la culture au Québec, Québec, Éditions Nota bene, 2004, 405 p.

+ CONFORTĖS, Claude, Répertoire du théâtre contemporain de langue française, Paris, F. Nathan, $447 \mathrm{p}$.

+ GLAAP, Albert-Reiner, Voices from Canada: Focus on 30 Plays, trad. à l'anglais par Nicholas P. Quaintmere, Toronto, Playwrights Canada Press, 142 p.

+ GREFFARD, Madeleine, et Jean-Guy SABOURIN, Le théâtre québécois, Montréal, Boréal, 120 p.

+ GREIF, Hans-Jürgen, et François OUELLET, La littérature québécoise, 1960-2000, Québec, L'Instant même, 2004, 116 p.

+ HAMEL, Réginald, Panorama de la littérature québécoise contemporaine, Montréal, Guérin, 1997, $822 \mathrm{p}$.

+ MAGNAN, Lucie-Marie, et Christian MORIN, 100 pièces du théâtre québécois qu'il faut lire et voir, Québec, Éditions Nota bene, 2002, 441 p.

\section{TRADUCTIONS DES EEURES DE MICHEL MARC B O U C H A R D 1}

\section{I . 1. Liv res}

+ Lilies or the Revival of a Romantic Drama [Les feluettes ou La répétition d'un drame romantique], trad. de Linda Gaboriau, Toronto, Coach House Press et Playwrights Canada Press, 1990. En anglais.

+ The Skelfs or The Rehearsal of a Romantic Drama [Les feluettes ou La répétition d'un drame romantique], trad. de Martin Bowman et Bill Findlay, inédit, 1993. En anglais pour l'Écosse.

+ "Le muse orfane [Les muses orphelines]», trad. de Francesca Moccagatta dans Il teatro del Québec, Milan, Ubulibri, 1994. En italien.

+ The Orphan Muses [Les muses orphelines], trad. de Linda Gaboriau, Winnipeg, Scirocco Drama, 1995. En anglais.

+ Desire [Le désir], trad. de Linda Gaboriau, Lac-Brome, inédit, 1996. En anglais.

+ Die Geschichte von Teeka [L'histoire de l'oie], trad. de Marie-Elisabeth Morf, édition à compte d'auteur, 1996. En allemand.

1 La recension de certains titres s'est heurtée à des problèmes d'accessibilité, notamment pour les «Traductions»; certaines références sont donc incomplètes. 
+ Heat Wave [Les grandes chaleurs], trad. de Bill Glassco, Winnipeg, Scirocco Drama, 1996. En anglais.

+ Pierre and Marie and the Devil with the Deep Blue Eyes [Pierre et Marie... et le démon], trad. de Linda Gaboriau, Lac-Brome, inédit, 1998. En anglais.

+ The Coronation Voyage [Le voyage du couronnement], trad. de Linda Gaboriau, Vancouver, Talonbooks, 1999. En anglais.

+ Die verlassenen Musen [Les muses orphelines], trad. de Frank Heibert, Reinbek [Allemagne], Rowohlt, 1999. En allemand.

+ The Tale of Teeka [L'histoire de l'oie], trad. de Linda Gaboriau, Vancouver, Talonbooks, 1999. En anglais.

+ Gefahrenzone [Le chemin des Passes-dangereuses], trad. de Frank Heibert, Reinbek [Allemagne], Rowohlt, 2000. En allemand.

+ Il cammino dei Passi Pericolosi : una tragedia stradale [Le chemin des Passes-dangereuses], Florence, Intercity Plays, 2000. En italien.

+ El camino de los pasos peligrosos [Le chemin des Passes-dangereuses] et Los Endebles o la Repetición de un drama romántico [Les feluettes ou La répétition d'un drame romantique], trad. de Boris Schoemann, Las musas huérfanas [Les muses orphelines], trad. de Natalia Traven, Mexico, Édiciones El Milagro, 2002. En espagnol pour le Mexique.

+ « Il Viaggio dell'incoronazione [Le voyage du couronnement] », «Il sentiero dei Passi Pericolosi [Le chemin des Passes-dangereuses] » et «Le Mammole, prova o ripetizione di un dramma romantico [Les feluettes ou La répétition d'un drame romantique]», trad. de Francesca Moccagatta; «I manoscritti del diluvio [Les manuscrits du déluge] » et «Il pittore di madonne o La nascita di un quadro di un quadro [Le peintre des madones ou La naissance d'un tableau]", trad. de Barbara Nativi dans Michel Marc Bouchard, Teatro, Milan, Ubulibri, 2003, 192 p. En italien.

+ «Muzele orfane [Les muses orphelines]», trad. de Aurel Stefanescu dans Musele Orfane, dramaturgie québechezã, Teatru de ieri si de azi, seria: Teatru Francofon, Bucarest, Éditura Viitorul Românesc, 2003. En roumain.

+ KOJI NO MUSE TACHI [Les muses orphelines], trad. de Ayako Sato, Tokyo, Sairyuusha, 2004. En japonais.

+ Night Butterflies [Les papillons de nuit], trad. de Linda Gaboriau, Lac-Brome, inédit, 2004. En anglais.

+ "Storia dell'oca [L'histoire de l'oie] », trad. de Francesca Moccagatta dans Intercity connections Nuovo testi per nuovi interpreti. Dieci testi teatrali per adolscenti, dir. par Rodolfo di Glammarco et Barbara Nativi, Rome, Editoria \& Spettacolo, 2004. En italien.

+ Written on Water [Les manuscrits du déluge], trad. de Linda Gaboriau, Vancouver, Talonbooks, 2004. En anglais.

+ El pintor de madonas o El nacimiento de un cuadro [Le peintre des madones ou La naissance d'un tableau], trad. de Álvaro Orrea, inédit, 2006. En espagnol.

+ Osirelé múzy [Les muses orphelines], trad. de Michal Lazovskych et Matylda Preklad, Prague, inédit, 2006. En tchèque.

+ Occhi di vetro [Des yeux de verre], trad. de Francesca Moccagatta, inédit, 2007. En italien.

+ Osierocone muzy [Les muses orphelines], trad. de Nicole Korzycka, Cracovie, inédit, 2007. En polonais.

\section{I. 2. En revue}

+ "Down Dangerous Passes Road [Le chemin des Passes-dangereuses]", trad. de Linda Gaboriau dans Canadian Theatre Review, nº 102, 2000, p. 48-68. En anglais. 


\section{PRIX ET D IS TINCTIONS}

+ Prix littéraire du Journal de Montréal 1988 pour Les feluettes.

+ Prix d'excellence du Cercle littéraire de l'Outaouais 1988 pour Les feluettes.

+ Prix Dora Moore 1991 pour Lilies.

+ Prix Chalmer 1991 pour Lilies.

+ Prix du Conseil des arts de Montréal 1992 pour L'histoire de l'oie.

+ Prix de l'Association québécoise des critiques de théâtre 1992 pour L'histoire de l'oie.

+ Prix du Meilleur spectacle étranger 1993 de l'Association des critiques de théâtre du Mexique pour La historia de la oca.

+ Prix du Centre National des Arts en 1993 avec la compagnie Les Deux Mondes pour la pièce L'histoire de l'oie.

+ Salamandre d'or à Blois en 1996 pour Lilies.

+ Prix du jury au Outfest Film Festival de Los Angeles 1996 pour Lilies.

+ Prix du meilleur film au Lesbian and Gay Film Festival de San Francisco 1996 pour Lilies.

+ Prix du public au Festival international d'Oslo 1996 pour Lilies.

+ Prix Génie 1996 du meilleur film canadien pour Lilies.

+ International Banff Rocke Award 1998 pour la meilleure émission jeunesse pour le téléfilm français L'histoire de l'oie et sa version anglaise The Tale of Teeka.

+ Prix Gémeau 1998 pour la meilleure émission jeunesse produite en français au Canada pour L'histoire de l'oie.

+ Golden Spire Award 1999 au San Francisco International Film Festival pour The Tale of Teeka.

+ Masque du public et Masque de la meilleure production montréalaise pour Les feluettes mise en scène par Serge Denoncourt à la Soirée des Masques 2003.

+ Membre de l'Ordre du Canada en 2005.

\section{V. RÉ C EPT IO N C R IT IQ UE}

\section{IV.1.1. Mémoires et $t$ hè se s}

+ BÉDARD, Ghislain, «Organisation spatiale et processus interactionnels dans Les feluettes de Michel Marc Bouchard», mémoire de maîtrise, Sainte-Foy, Université Laval, 1994, 142 f.

+ COURCHESNE, Lucie, «Le mélodramatique dans Aurore l'enfant martyre, Les oranges sont vertes et Les feluettes », mémoire de maîtrise, Montréal, Université de Montréal, 2002, 133 f.

+ DUGUAY, Sylvain, «Le dialogue homosexuel dans Les feluettes de Michel Marc Bouchard», mémoire de maîtrise, Montréal, Université McGill, 1999, 99 f.

+ EPISALE, Frank, «Character-driven: Sexual Delinquents in M. Butterfly and Lilies», in " (Meta)theatrical Representations of Imprisonment in Contemporary Drama», thesis for the degree of Master of Arts in Theatre, Hawaii, University of Hawaii, 2006, f. 43-62.

+ HUFFMAN, Shawn, «L'Affect en cachot. La sémiotique des passions et le théâtre québécois d'enfermement chez Michel Marc Bouchard, Normand Chaurette et René-Daniel Dubois ", thèse de doctorat, Toronto, University of Toronto, 1998, $409 \mathrm{f}$.

+ KENZIE, Meredith, "Chapter Four: Image and Metaphor in The Tale of Teeka ", in "Breaking Barriers. Using Theatre with Teenage Audiences to Communicate about Sex, Drugs and Violence», mémoire de maîtrise, Edmonton, University of Alberta, 2006, f. 81-99.

+ LESSARD, Maude, «L'écriture du masque. Formes et fonctions de la surthéâtralisation dans l'œuvre de Michel Marc Bouchard», mémoire de maîtrise, Montréal, Université du Québec à Montréal, 2001, 109 f. 
+ LEVASSEUR, André, «Palimpsexes, le genre au second degré. La construction intertextuelle et parodique du travestissement dans Les muses orphelines de Michel Marc Bouchard", mémoire de maîtrise, Montréal, Université du Québec à Montréal, 1996, 86 f.

+ PAPACHRISTOS, Katherine, «Le théâtre dans le théâtre. Étude du fonctionnement du procédé du théâtre dans le théâtre chez certains dramaturges contemporains», mémoire de maîtrise, Sainte-Foy, Université Laval, 1987.

+ PLOURDE, Mélanie, «Mettre l'écriture en scène. L'autoreprésentation dans la dramaturgie québécoise des années quatre-vingt ", mémoire de maîtrise, Montréal, Université du Québec à Montréal, 2000, $124 \mathrm{f}$.

+ TREMBLAY, Janie, «Les muses orphelines», dans «Le personnage de la mère dans trois pièces québécoises des années 1980 ", mémoire de maîtrise, Montréal, Université McGill, 2002, f. 6382.

\section{IV.1.2. Parties d'ouvrages}

+ ANDRĖS, Bernard, et Pascal RIENDEAU, «La dramaturgie depuis 1980 », Panorama de la littérature québécoise contemporaine, sous la dir. de Réginald Hamel, Montréal, Guérin, 1997, p. 208-239.

+ DEFRAEYE, Piet, et Marylea MACDONALD, "Les feluettes, un drame de répétition », Nouveaux regards sur le théâtre québécois, sous la dir. de Betty Berdnardski et Irène Oore, Montréal, XYZ éditeur, 1997, p. 129-137.

+ GODIN, Jean-Cléo, et Dominique LAFON, «La contre-nature de Michel Marc Bouchard, dramaturge du terroir ", Dramaturgies québécoises des années quatre-vingt: Michel Marc Bouchard, René-Daniel Dubois, Marie Laberge, Montréal, Leméac, 1999, p. 61-102.

+ O'NEILL-KARCH, Mariel, «Espace-théâtre dans Les feluettes », Théâtre franco-ontarien: espaces ludiques, Vanier, L'Interligne, 1992, p. 121-138.

+ RIENDEAU, Pascal, «Le théâtre des feluettes ou la lecture romantique de Michel Marc Bouchard», Texte et représentation: les arts du spectacle (XVI $I^{e}$ XVIII siècles), Texte, nos 33/34, 2003, p. 289-310.

+ WALLACE, Robert, «Reflections on Critical Responses to Francophone Theatre in Toronto: January 1986 - November 1988 ", Producing Marginality, Theatre and Criticism in Canada, sous la dir. de Robert Wallace, Saskatoon [Saskatchewan], Fifth House Pub, 1990, p. 213-242.

\section{V. 1 . 3. É t u d e s}

+ [Collectif], «Théâtre et homosexualité», Jeu, n 54, 1990 (mars), p. 5-133.

+ BELZIL, Patricia, «Des muses inspirantes? », Jeu. Théâtre et cinéma, nº 98, 2001 (mars), p. 172. [film]

+ BÉNARD, Johanne, «Écouter le théâtre [sur disque compact] », Jeu, no 84, 1997 (septembre), p. 24-28.

+ BLONDE, David, «Entre Oreste et Barbe-Bleue: la violence dans la scène familiale québécoise, 1981-2002", L'Annuaire théâtral, no 32, 2002 (automne), p. 129-149.

+ BOURASSA, André-G., «Entre EEdipe et Pygmalion», Lettres québécoises, n 43, 1986 (automne), p. 38-39.

+ BOURASSA, André-G., «Cachez ce saint... ou l'émergence, dans notre espace dramatique, de saint Jean-Baptiste, saint Laurent, saint Sébastien, ainsi soient-ils!», Lettres québécoises, no 49, 1988 (printemps), p. 49. 
+ ÉTHIER, Jean René, «Les feluettes ou la déviance thêâtrale», L'Analyste, n 25, 1989, p. 79-81.

+ GOBERT, R. Darren, «The Antitheatrical Paradox in Michel Marc Bouchard's Les Feluettes, ou la répétition d'un drame romantique», Canadian Literature, nº 188, 2006 (printemps), p. 47-61.

+ GRAEFE, Sara, «Reviving and Revising the Past: The Search For Present Meaning Michel Marc Bouchard's Lilies, or The Revival of a Romantic Drama», Theatre Research in Canada/Recherches théâtrales du Canada, vol. 14, no 1 (printemps), 1993, p. 165-177.

+ HUFFMAN, Shawn, «Le trépas vient tout guérir? Saint Sébastien dans Les feluettes de Michel Marc Bouchard", Synthèses, vol. 1, nº 1, s. d., p. 57-73.

+ HUFFMAN, Shawn, «Dans les coulisses de la fiction: le dramaturge préfacier dans le thêâtre québécois contemporain", L'Annuaire théâtral, nº 34, 2003 (automne), p. 58-80.

+ LAFON, Dominique, «Les muses orphelines [pièce de Michel Marc Bouchard] », Jeu, n 74, 1995 (mars), p. 79-88.

+ LAFON, Dominique, «Nébuleuse créatrice et constellation familiale: les trois pièces satellites du Chemin des Passes-dangereuses, de Michel Marc Bouchard", L'Annuaire théâtral, n 27, 2000 (printemps), p. 191-203.

+ LÉVESQUE, Solange, «Éclaircie dans l'été des théâtres », Jeu, n 85, 1997 (décembre), p. 167170.

+ LÉVESQUE, Solange, et Diane PAVLOVIC, «Comédiens et martyrs», Jeu, n 49, 1988 (décembre), p. 152-167.

+ LÉVESQUE, Solange, Diane PAVLOVIC et Isabelle RAYNAULD, «Les feluettes ou La répétition d'un drame romantique», Jeu, no 49, 1988 (décembre), p. 151-179.

+ LOISELLE, André, «Le cadavre à l'intersection du théâtre et du cinéma dans Lilies et Being at home with Claude», L'Annuaire théâtral, nº 30, 2001 (automne), p. 75-93.

+ LOISELLE, André, «Les muses orphelines du théâtre au cinéma : en conversation avec Robert Favreau ", L'Annuaire théâtral, nº 30, 2001 (automne), p. 97-106.

+ LOISELLE, André, " "Translation" from Theatre to the Cinema: Some Examples of Canadian Repertoire», Theatre Research in Canada/Recherches thêâtrales au Canada, vol. 24, nos 1-2, 2003 (printemps/automne), p. 21-38.

+ MOSS, Jane, "Sexual Games: Hypertheatricality and Homosexuality in recent Quebec Plays», The American Review of Canadian Studies, vol. 17, n 3, 1987 (automne), p. 287-296.

+ ROCHELEAU, Alain-Michel, et Luke STANDFORD, «Gay Theater in Quebec: The Search for an Identity", Yale French Studies, nº 90, 1996, p. 115-136.

+ TEISSIER, Guy, "Ā la recherche de la parole. Le thêâtre de Michel Marc Bouchard», Revue d'histoire du théâtre, nº 202, 1999, p. 5-20.

+ TEMKINE, R. "An Absent, but still Very Present, Father (Common Theme in Plays by JeanClaude Grumberg, Michel Marc Bouchard, and Michael Frayn)», Europe, vol. 77, n 840, 1999 (avril), p. 269-274.

\section{IV.1.4. Commentaires parus dans des périodiques}

+ BEAUCAGE, Christian, "Thêâtre et homosexualité: prendre part à la différence», Québec français, nº 124, 2001-2002 (hiver), p. 38-42.

+ BOURASSA, André-G., «Hold up», Lettres québécoises, no 49, 1988 (printemps), p. 51.

+ GODIN, Jean-Cléo, et Dominique LAFON, «Bouchard, Dubois, Chaurette», Théâtre/Public. Théâtre - Québec (du texte à la scène), nº 117, 1994, p. 49-53.

+ LAVOIE, Pierre, «De la rigueur et de la susceptibilité», Jeu, nº 67, 1993 (juin), p. 131-135. 
+ LÉPINE, Stéphane, "La Contre-nature de Chrysippe Tanguay, écologiste. Récréation et engendrement: quelques considérations psychodramatiques », Jeu, no 32, 1984, p. 137-140.

+ SIRARD, Pascale, «Les muses orphelines de Michel Marc Bouchard», Spirale, n 139, 1995 (février), p. 19.

\section{IV.2. Comptes rendus et articles de presse}

\section{IV.2.1. La contre-nature de Chrysippe Tanguay}

+ DiONNE, André, «Le théâtre qu'on joue», Lettres québécoises, n 33, 1984 (printemps), p. 6768.

+ DONNELLY, Pat, [sans titre], The Gazette, 18 septembre 1987, p. C8.

+ FRASER, Matthew, «Les Feluettes returns in Triumph. A Work of Power, Imagination », The Globe and Mail, 30 novembre 1987, p. C9.

+ PELLETIER, Francine, «Un théâtre de nouveaux gais», La Vie en rose, nº 15, 1984 (janvierfévrier), p. 60.

+ ROBERT, Lucie, «La passion du dialogue», Voix et Images, vol. 11, n 1, 1985 (automne), p. $140-145$.

\section{IV.2.2. La poupée de Pélopia}

+ DIONNE, André, «Le théâtre qu'on joue», Lettres québécoises, n 37, 1985 (printemps), p. 5557.

+ ROBERT, Lucie, «La littêrature au théâtre », Voix et Images, vol. 12, n 1, 1986 (automne), p. $155-160$.

\section{IV.2.3. Rock pour un faux-bourdon}

+ ROBERT, Lucie, "Amours, délices et orgues», Voix et Images, vol. 13, nº 2, 1988 (hiver), p. 357359.

\section{IV.2.4. Les feluettes}

+ [Anonyme], «Les feluettes», Le Soleil, 18 septembre 2004, p. C13.

+ [Anonyme], «Les feluettes», Le Soleil, 24 septembre 2004, p. B2.

+ [Anonyme], "Lilies, or the Revival of a Romantic Drama», Canadian Theatre Review, n 72 , 1992 (automne), p. 79-81.

+ BELZIL, Patricia, «Les feluettes», Jeu, n 52, 1989 (septembre), p. 211.

+ BELZIL, Patricia, "Lilies ou la répétition d'un nouveau drame romantique», Jeu, no 83, 1997 (juin), p. 174-178. [film]

+ BILODEAU, Martin, «Michel Marc Bouchard gagne le pari de la transposition», Le Devoir, 30 août 1996, p. A1 et A10. [film]

+ BILODEAU, Martin, «Un événement digne des Feluettes», Le Devoir, 26 octobre 1996, p. B6. [film]

+ BOULANGER, Luc, "Les feluettes», Voir, 21 novembre 2002, p. 48.

+ COMTOIS, Mélissa, «Au-delà des murs, la mémoire... », Jeu, nº 115, 2005 (juin), p. 76-79.

+ DONNELLY, Pat, «Homosexual Passion, Religious Theatre Crux of Les Feluettes», The Gazette, 12 septembre 1987, p. D13.

+ ÉTHIER, Jean-René, et Jean-Noël TREMBLAY, «Les feluettes ou la déviance théâtrale», L'Analyste, n 25, 1989 (printemps), p. 79-81.

+ FOURLANTY, Éric, «Violence et passion», Voir, 24 octobre 1996, p. 31. [film] 
+ GRIFFIN, John, «Lilies Polished to Near-perfection in Sight and Sound», The Gazette, 25 octobre 1996, p. D3.

+ HÉTU, Pierre, «Les feluettes, Michel Marc Bouchard», Nuit blanche, nº 34, 1988/1989 (décembre-janvier-février), p. 14.

+ KOUSTAS, Jane, "[Lilies or the Revival of a Romantic Drama, trad. Linda Gaboriau] », University of Toronto Quarterly, vol. 62, nº 1, 1992 (hiver), p. 115.

+ LÉPINE, Stéphane, «Le théâtre qu'on joue», Lettres québécoises, n 48, 1987/1988 (hiver), p. $42-44$.

+ LÉVESQUE, Robert, «Bouchard et les feux de Roberval», Le Devoir, 25 septembre 1987, p. 13.

+ L'HÉRAULT, Pierre, «Enregistrer le mouvement», Spirale, nº 189, 2003 (mars-avril), p. 54-55.

+ MCLAREN, Leah, "Out of the Closet and Onto the Screen», The Globe and Mail, 12 août 1999, p. C1. [film]

+ PORTER, Isabelle, «L'autre Frédéric Dubois», Le Devoir, 27 septembre 2004, p. B8.

+ POULIOT, Sophie, «Intense histoire d'amour», Le Devoir, 16 novembre 2002, p. E4.

+ QUIROT, Odile, «Haute surveillance. La religion et l'homosexualité: Les feluettes ou la répétition d'un drame romantique du Québécois Michel Marc Bouchard», Le Monde, 31 janvier 1990, p. 19.

+ SAINT-PIERRE, Christian, «Le vrai du faux», Jeu, nº 107, 2003 (juin), p. 76-81.

\section{IV.2.5. Les muses orphelines}

+ [Anonyme], «Vent de folie familiale au Théâtre des Osses», Le Temps, 11 décembre 2002, n. p.

+ BEAUNOYER, Jean, «Les muses orphelines : une œuvre de maturité», La Presse, 11 septembre 1988, p. E1.

+ BONHAM, R. A., «The Orphan Muses », Canadian Theatre Review, n 86, 1996 (printemps), p. 63-65.

+ BOUVET, Bruno, «Les muses orphelines trouvent un joli refuge», La Croix, 3 janvier 2005, p. 24.

+ BRÉDY, Aude, «Famille, tu me déchires, tu me mens», L'Humanité, 20 juillet 2004, p. 20.

+ CARON, Catherine, Lucie ROBERT et Jean-Louis TREMBLAY, "Coups d'œil [sur quelques pièces de thêâtre]", Jeu, nº 58, 1991 (mars), p. 196-199.

+ CASTIEL, Elie, et Pierre RANGER, «Les muses orphelines [de Robert Favreau] », Séquences, n² 209, 2000 (septembre-octobre), p. 32-36. [film]

+ CONLOGUE, Ray, "Les muses orphelines», The Globe and Mail, 28 novembre 1994, p. C1.

+ COPPERMANN, Annie, «Le poids de l'abandon», Les Échos, 14 décembre 2004, p. 15.

+ CÔTÉ, Christian, «Les muses orphelines : un bijou venu du nord», Le Droit, 7 novembre 2000, p. 39. [film]

+ DONNELLY, Pat, «Dysfunctional Family, Quebec-style, makes for fine Drama. Les Muses Orphelines Superbly Acted, Directed», The Gazette, 21 septembre 1995, p. C7.

+ DREYFUS, Alain, «Appartement agrandi d'une pièce», Libération, 24 novembre 2003, p. 37.

+ HÉLIOT, Armelle, «Les enfants perdus», Le Figaro, 8 décembre 2004, p. 27.

+ KOUSTAS, Jane, «[The Orphan Muses, trad. Linda Gaboriau]» University of Toronto Quarterly, vol. 66, no 1, 1996/1997 (hiver), p. 115.

+ LAVOIE, André, "Château en Espagne», Le Devoir, 2 juillet 2005, p. 24.

+ LÉPINE, Stéphane, «Le théâtre qu'on joue», Lettres québécoises, nº 52, 1988/1989 (hiver), p. $46-48$. 
+ LÉVESQUE, Robert, «La métamorphose de la mongole», Le Devoir, 13 septembre 1988, p. 9.

+ LÉVESQUE, Robert, «Saint-Ludger-de-Milot à Manhattan», Le Devoir, 7 décembre 1993, p. B8.

+ LÉVESQUE, Robert, «La résurrection d'une pièce», Le Devoir, 20 octobre 1994, p. B8.

+ LÉVESQUE, Solange, «Des Muses inspirantes», Le Devoir, 3 novembre 1997, p. B8.

+ LIBAN, Laurence, «Les muses orphelines», L'Express, 21 février 2005, p. 102.

+ LUSSIER, Marc-André, "Les muses orphelines», La Presse, 4 novembre 2000, p. C2. [film]

+ MECHLING, Lauren, "A Dysfunctional Family Reunion", National Post, 9 mars 2001, p. B4.

+ PROVENCHER, Normand, "Critique», Le Soleil, 4 novembre 2000, p. G2. [film]

+ RENAULT, Gilles, "“Muses" de compassion», Libération, 9 avril 2005, p. 38.

+ SÉLIGNAN, Maïté, «La complainte de la belle province», Le Figaro, 4 janvier 2005, n. p.

+ TREMBLAY, Odile, «Une fort agréable surprise», Le Devoir, 4 novembre 2000, p. C6. [film]

+ VIGEANT, Louise, «Les muses orphelines», Jeu, no 49, 1988 (décembre), p. 197-199.

\section{IV.2.6. Soirée bénéfice pour ceux qui ne seront pas là en l'an 2000}

+ CONLOGUE, Ray, "The Fruits of "Spitting Poison"», The Globe and Mail, 6 juin 1992, p. C15.

+ LÉVESQUE, Robert, «Michel Marc Bouchard dans la gueule du loup », Le Devoir, 6 juin 1992, p. A1 et A4.

\section{IV.2.7. L'histoire de l'oie}

+ [Anonyme], «L'histoire de l'oie», L'Express, 28 octobre 1999, p. 54.

+ [Anonyme], «The Tale of Teeka», Canadian Literature, n 167, 2000 (hiver), p. 180.

+ DAVID, Gilbert, «Walt Disney chez Kafka», Le Devoir, 23 novembre 1991, p. C5.

+ DELGADO, Jérôme, "L'oie sans frontières», La Presse, $1^{\text {er }}$ octobre 2005, p. 5 (Arts et spectales).

+ KOUSTAS, Jane, «The Tale of Teeka, trad. Linda Gaboriau» University of Toronto Quarterly, vol. 70, nº 1, 2000 (hiver), p. 261.

+ LABRECQUE, Marie, «L'histoire de l'oie», Voir, 17 avril 1997, p. 61.

+ LACEY, Liam, "The Tale of Teeka», The Globe and Mail, 18 juin 1992, p. E3.

+ LÉVESQUE, Robert, «Le théâtre tout court», Le Devoir, 28 novembre 1991, p. B3.

+ POPOVIC, Pierre, Hélène RICHARD et Nadine VINCENT, «Spectacles: critiques», Jeu, n 62, 1992 (mars), p. 155-162.

+ TAYLOR, Kate, "The Tale of Teeka», The Globe and Mail, 17 mai 1995, p. C4.

+ TURCOT, Geneviève, «Il était une fois la violence... », Le Droit, 5 février 2005, p. A13.

+ VAIS, Michel, «Les Feluettes mexicaines et autres étrangetés», Jeu, nº 118, 2006 (mars), p. 121125.

\section{IV.2.8. Les grandes chaleurs}

+ BÉLAIR, Michel, «Éloge de la différence», Le Devoir, 4 juillet 1994, p. B7.

+ KNOWLES, Richard Paul, «[Heat Wave], University of Toronto Quarterly, vol. 67, n 1, 1997/1998 (hiver), p. 71.

+ LECOMPTE, Anne-Marie, "Les grandes chaleurs : un divertissement intelligent», La Presse, 10 juillet 1993, p. D2.

+ O'NEILL-KARCH, Mariel, «[Les grandes chaleurs]», University of Toronto Quarterly, vol. 64, $\mathrm{n}^{\circ}$ 1, 1994 (hiver), p. 111.

+ ST-HILAIRE, Jean, «Les grandes chaleurs », Le Soleil, 12 juillet 2003, p. C8. 


\section{IV.2.9. Le voyage du couronnement}

+ BLAGRAVE, Mark, «Michel Marc Bouchard, The Coronation Voyage - Translated from Québécois ", Canadian Literature, n 175, 2002 (hiver), p. 116-118.

+ BOULANGER, Luc, «La folie du large», Voir, 14 septembre 1995, p. 12.

+ CONLOGUE, Ray, «Le voyage du couronnement», The Globe and Mail, 28 septembre 1995, p. C2.

+ CUSHMAN, Robert, "Heavy is the Head that Goes to See the Crown", National Post, 19 septembre 2000, p. B16.

+ DAVID, Gilbert, «Michel Marc Bouchard face aux fils sacrés», Le Devoir, 16 septembre 1995, p. B6.

+ DONNELlY, Pat, "Voyage is an Overburdened Vessel Buoyed up by Good Performances», The Gazette, 23 septembre 1995, p. D7.

+ GODIN, Jean-Cléo, Hélène RICHARD et Marie-Christine LESAGE, "Spectacles: critiques », Jeu, n 77, 1995 (décembre), p. 199-209.

+ LÉVESQUE, Robert, «Un bateau que Dieu sait qui...», Le Devoir, 26 septembre 1995, p. B8.

+ O'NEILL-KARCH, Mariel, "[Le voyage du couronnement]", University of Toronto Quarterly, vol. 66, n 1, 1996/1997 (hiver), p. 40.

+ ROBERT, Lucie, «Minces propos», Voix et Images, vol. 23, n 1, 1997 (automne), p. 181-188.

+ ST-HILAIRE, Jean, «Le voyage du couronnement», Le Soleil, 21 novembre 1995, p. B7.

+ TAYLOR, Kate, "Abandon Ship - This one's Sinking Fast», The Globe and Mail, 8 février 2000, p. R3.

+ TAYLOR, Kate, «A Grand and Masterful Sail», The Globe and Mail, 8 juillet 2003, p. R3.

+ YOUNG, David, «Critics, Take a Bow», The Globe and Mail, 5 décembre 2001, p. R3.

\section{V.2.10. Le désir}

+ GUAY, Hervé, «Une comédie alimentaire», Le Devoir, 20 juin 1996, p. B7.

+ ST-HILAIRE, Jean, «Le désir», Le Soleil, 19 juillet 1996, p. B10.

\section{IV.2.11. Une entreprise amoureuse}

+ BEAUNOYER, Jean, «Une entreprise amoureuse réussie», La Presse, 2 juillet 1996, p. B9.

\section{IV.2.12. Les aboyeurs}

+ [Anonyme], «Féroce», Le Soleil, 9 juillet 1999, p. B1.

+ CANTIN, David, «Juste portrait de ces "aboyeurs médiatiques"», Le Devoir, 9 août 1999, p. B8.

\section{IV.2.13. Le chemin des Passes-dangereuses}

+ [Anonyme], «Down Dangerous Passes Road», Canadian Literature, 2003 (été), p. 130.

+ BARRIĖRE, Caroline, «Un huis clos en pleine forêt», Le Droit, 21 février 2002, p. 40.

+ DONNELLY, Pat, «Road Tragedy Crashes, much too Slowly», The Gazette, 3 mars 1998, p. B5.

+ KOUSTAS, Jane, «[Down Dangerous Passes Road, trad. Linda Gaboriau]» University of Toronto Quarterly, vol. 71, nº 1, 2001/2002 (hiver), p. 98.

+ MAKEREL, Catherine, "Aux frontières de l'illusion", Le Soir, 19 août 2005. n. p.

+ O'NEILL-KARCH, Mariel, "Theatre [Le chemin des Passes-dangereuses] », University of Toronto Quarterly, vol. 69, n 1, 1999/2000 (hiver), p. 69.

+ PERRIER, Jean Louis, «Randonnée sur les sentes tragiques du souvenir», Le Monde, 20 février 1999, p. 32. 
+ RIENDEAU, Pascal, «Choc fraternel», Jeu, nº 87, 1998 (juin), p. 39-42.

+ RIVERS, Paul, et Jim WONG-CHU, «Familiar Geography. Looking for Home Down Dangerous Passes Road ", Canadian Theatre Review, no 110, 2002 (printemps), p. 96.

\section{IV.2.14. Pierre et Marie... et le démon}

+BARRIËRE, Caroline, «Les hauts et les bas de la vie d'un couple sans histoire», Le Droit, 5 juillet 2003, p. A7.

+ CHAREST, Rémy, «Gentiment subversif», Le Devoir, 7 juillet 1998, p. B8.

+ DONNELLY, Pat, "Bisexuality adds Spice to Bedroom-farce Routine», The Gazette, 10 août 1998, p. B5.

+ ST-HILAIRE, Jean, «Pierre et Marie... et le démon», Le Soleil, 12 juin 1998, p. C12.

\section{IV.2.15. Les papillons de nuit}

+ LÉVESQUE, Solange, "Chassé-croisé amoureux», Le Devoir, 21 juillet 1999, p. B8.

+ O’NEILL-KARCH, Mariel, "[Les papillons de la [sic] nuit]», University of Toronto Quarterly, vol. 70, nº 1, 2000 (hiver), p. 71.

\section{IV.2.16. Sous le regard des mouches}

+ [Anonyme], «Sous le Regard is Worth a Look», The Gazette, 3 mars 2000, p. D10.

+ BAILlARGEON, Stéphane, "Sa majesté des mouches", Le Devoir, 12 février 2000, p. B3.

+ DONNELlY, Pat, «Pigs, Flies and other Farm Oddities. Playwright Bouchard Turns Director for a Creepy Comedy set in a Mansion cum Abattoir», The Gazette, 24 février 2000, p. D9.

+ GODIN, Jean-Cléo, "Des cochons et des hommes», Jeu, n 95, 2000 (juin), p. 17-18.

+ SAFARTI, Sonia, «Sous le regard des mouches», La Presse, 24 février 2000, p. E6.

\section{IV.2.17. Les manuscrits du déluge}

+ AL-SOLAYLEE, Kamal, «Loitering Elegantly, with no Clear Intent», The Globe and Mail, 24 janvier 2004, p. R10.

+ BARTON, Bruce, «[Written on Water] », University of Toronto Quarterly, vol. 74, nº 1, 2004/2005 (hiver), p. 271-273.

+ BELLIVEAU, Georges, «Translating Drama», Canadian Literature, n 187, 2005 (hiver), p. 153-155.

+ CUSHMAN, Robert, «A Drama that Threatens to go Overboard», National Post, 9 juillet 2003, p. AL7.

+ DUMAS, Ėve, «Souvenirs submergés», La Presse, 8 février 2003, p. D3.

+ DUMAS, Ève, «Un Michel Marc Bouchard dilué», La Presse, 15 février 2003, p. D7.

+ GUAY, Hervé, «Mission impossible», Le Devoir, 17 février 2003, p. B8.

+ HÉBERT, Catherine, «Michel Marc Bouchard et Barbara Nativi», Voir, 6 février 2003, p. 34.

+ HÉLIOT, Armelle, «Un flottement certain. Les manuscrits du déluge de Michel Marc Bouchard au Tristan Bernard ", Le Figaro, 11 septembre 2006, p. 36.

+ LABRECQUE, Marie, «Les manuscrits du déluge», Voir, 20 février 2003, p. 40.

+ LANE-MERCIER, Gillian, et Agnes WITHFIELD, «[Written on Water]», University of Toronto Quarterly, vol. 75, nº 1, 2006 (hiver), p. 131.

+ LIBAN, Laurence, «Les manuscrits du déluge», L’Express, n² 2881, 21 septembre 2006, p. 102.

+ O’NEILL-KARCH, Mariel, «[Les manuscrits du déluge]», University of Toronto Quarterly, vol. 75, $n^{\circ} 1,2004 / 2005$ (hiver), p. 68. 
+ POPOVIC, Pierre, "Quand la mémoire prend l'eau», Jeu, nº 108, 2003 (septembre), p. 8-11.

+ POSNER, Michael, "The Tale of a Playwright on Rough Water», The Globe and Mail, 27 janvier 2004, p. R3.

+ RADZ, Matt, «Grandeur but not Greatness», The Gazette, 20 février 2003, p. D4.

+ RADZ, Matt, «Hope After the Deluge», The Gazette, 17 avril 2004, p. H1.

IV.2.18. Le peintre des madones ou La naissance d'un tableau

+ BARRIÈRE, Caroline, "Le peintre des madones ou la grande noirceur», Le Droit, 7 février 2005, p. 21.

+ BÉRUBÉ, Jade, «Le peintre des madones», Voir, 15 avril 2004, p. 21.

+ DUMAS, Ève, «Foisonnante fresque», La Presse, 10 avril 2004, p. 5 (Arts et spectacles).

+ GODIN, Jean-Cléo, «La Madone avortée et le chérubin difforme», Jeu, nº 112, 2004 (septembre), p. 22-23.

+ LÉVESQUE, Solange, "Au carrefour de l'amour et du sacré», Le Devoir, 3 avril 2004, p. E3.

+ LÉVESQUE, Solange, "Le peintre des madones», Le Devoir, 15 janvier 2005, p. E3.

+ O'NEILL-KARCH, Mariel, "[Le peintre des madones ou la naissance d'un tableau] », University of Toronto Quarterly, vol. 74, n 1, 2006 (hiver), p. 525.

+ POULIOT, Sophie, "De chair et de sang», Le Devoir, 10 avril 2004, p. C9.

+ ROBERT, Lucie, "À l'origine de toutes les origines», Voix et Images, vol. 31, n 3, 2006 (printemps), p. 146-156.

+ ST-HILAIRE, Jean, «Après la chute», Le Soleil, 11 février 2005, p. B5.

+ TURCOT, Geneviève, «Un conte érotico-religieux de Michel Marc Bouchard», Le Droit, 2 février 2005, p. 42.

\section{IV.2.19. Les porteurs d'eau}

+ O’NEILL-KARCH, Mariel, «[Les porteurs d'eau]», University of Toronto Quarterly, vol. 74, nº 1, 2006 (hiver), p. 525.

\section{V.2.20. Des yeux de verre}

+ CADIEUX, Alexandre, «Tragédie dans une maison de poupée», Le Devoir, 19 avril 2007, p. B7.

+ LABRECQUE, Marie, «Les ravages du déni», Le Devoir, 7 avril 2007, p. E3.

+ ST-JACQUES, Sylvie, «L'histoire d'une poupée brisée», La Presse, 16 avril 2007, p. 5 (Arts et spectacles).

+ ST-PIERRE, Christian, «Marie-Thérèse Fortin. Maison de poupée», Voir, 5 avril 2007, p. 16.

+ ST-PIERRE, Christian, «Des yeux de verre», Voir, 19 avril 2007, p. 16.

\section{IV.2.21. La nouvelle fabuleuse ou Les aventures d'un Flo}

+ [Anonyme], «Dans les coulisses de La fabuleuse», Le Soleil, 6 juin 2007, p. A1.

+BEAUNOYER, Jean, "Grandiose avec de l'émotion», La Presse, 2 mai 2007, p. 5 (Arts et spectacles).

+ MARIN, Stéphanie, "La fabuleuse se refait une beauté», Le Devoir, 2 mai 2007, p. B9.

+ TREMBLAY, Régis, «Métamorphosée, mais toujours démesurée», Le Soleil, 1 ${ }^{\mathrm{er}}$ mai 2007, p. A2. 


\section{IV.3. SUR ET AUTOUR DE L'AUTEUR}

\section{IV.3.1. A r ticles}

+ [Anonyme], «Michel Marc Bouchard sans frontières», Le Devoir, 18 juillet 1996, p. B7.

+ BAILLARGEON, Stéphane, "Le dramaturge globe-trotter», Le Devoir, 2 juillet 1996, p. B1.

+ BOULANGER, Luc, «Michel Marc Bouchard», Voir, 10 février 2000, p. 52.

+ CONLOGUE, Ray, «Quebec Theatre's Bright New Hope IN PERSON/As he Ventures into Film, Michel Marc Bouchard Keeps a Firm Grip on his Baroque Side», The Globe and Mail, 7 octobre 1995. p. C1.

+ DUCHARME, André, "Théâtre pour deux», L'Actualité, vol 20, nº 14, 15 septembre 1995, p. 88-92.

+ LAURIN, Danielle, «Après lui le déluge!», L'Actualité, vol. 28, n 3, $1^{\mathrm{er}}$ mars 2003, p. 64-68.

+ LEEVESQUE, Robert, "Michel Marc Bouchard: Roberval, 1912, j'avais 19 ans... », Le Devoir, 12 septembre 1987, p. C1-C2.

+ MORISSETTE, Brigitte, «Michel Marc Bouchard livre aux Mexicains quelques secrets de son succès au thêâtre», La Presse, 30 juillet 2001, p. C2.

\section{V.3.2. Entrevues}

+ BÉLAIR, Michel, «Fragments dans le désordre», Le Devoir, 8 février 2003, p. E3.

+ BELZIL, Patricia, «Contraintes et libertés de la scénarisation. Entretien avec Michel Marc Bouchard», Jeu, n 88, 1998 (septembre), p. 46.

+ BOIRON, Chantal, «Les feluettes de Michel Marc Bouchard: l'insolite de la passion. Entretien avec Michel Marc Bouchard», Acteurs Auteurs, nos 75-76, 1990, p. 43.

+ BOIRON, Chantal, «L'hiver au théâtre, c'est étrange, nous ne rions pas. Entretien avec Michel Marc Bouchard», Prospero, n 2, 1992 (juillet), p. 22.

+ BOUCHARD, Geneviève, "Une bête à plusieurs têtes», Le Soleil, 23 décembre 2006, p. A4.

+ BOULANGER, Luc, «Michel Marc Bouchard», Voir, 13 octobre 1994, p. 46.

+ CLOUTIER, Anne-Marie, «Michel Marc Bouchard», La Presse, 3 avril 2004, p. 3 (Arts et spectacles).

+ DIONNE, André, «Michel Marc Bouchard: tout plein d'émotions», Lettres québécoises, n 53 , 1989 (printemps), p. 10-13.

+ GODARD, Colette, «L'Été festival Avignon. Dernières nouvelles de nos cousins invités par l'Association française d'action artistique, deux auteurs québécois sont venus à Avignon [Entretien avec Michel Marc Bouchard et Jean-Marc Dalpé] », Le Monde, lundi 27 juillet 1992, p. 15.

+ HÉBERT, Catherine, "Michel Marc Bouchard et Barbara Nativi», Voir, 6 février 2003, p. 33.

+ LESAGE, Marie-Christine, "Michel Marc Bouchard: entre le rêve et la tourmente», Nuit blanche, n 61, 1995 (automne), p. 15-18.

+ LÉVESQUE, Solange, «Michel Marc Bouchard: la parole acérée», Le Devoir, 13 mars 1999, p. E22.

+ PARÉ, Yvon, «Cercle de presse du Saguenay», Le Quotidien, 2 octobre 2004, p. 42.

+ PETROWSKI, Nathalie, «Michel Marc Bouchard», La Presse, 17 juillet 2000, p. A1.

+ PROUVOST, Christelle, «Le chant des muses québécoises, rencontre avec Michel Marc Bouchard, auteur de thêâtre, associant qualité et succès », Le Soir, Bruxelles, 3 juillet 2006, p. 31.

+ ST-HILAIRE, Jean, «Les Pâques d'Isabelle», Le Soleil, 19 septembre 2006, p. A5. 
+ VAIS, Michel, «Peut-on former un auteur de théâtre? Les entrées libres de Jeu », Jeu, n 120, 2006 (juin), p. 82-95.

\section{V.3.2. Af f i c h e s}

+ La contre-nature de Chrysippe Tanguay, mise en scène par André Brassard, Montréal, Théâtre d'Aujourd'hui, 1983.

+ Les manuscrits du déluge, mise en scène par Barbara Nativi, Montréal, Théâtre du Nouveau Monde (TNM), 2003.

\section{V.3 . 4. Prog ra m m e s}

+ La contre-nature de Chrysippe Tanguay, mise en scène par André Brassard, Montréal, Théâtre d'Aujourd'hui, 1983.

+ La poupée de Pélopia, mise en scène par Michèle Magny, Montréal, Théâtre d'Aujourd'hui, 1984 (novembre).

+ Le chemin des Passes-dangereuses: une tragédie routière, mise en scène par Serge Denoncourt, Montréal, Compagnie Jean Duceppe, 1988 (février).

+ Les feluettes ou la répétition d'un drame romantique, mise en scène par André Brassard, Montréal, Théâtre du Nouveau Monde (TNM), 1989 (janvier).

+ L'histoire de l'oie, mise en scène par Daniel Meilleur, Montréal, Théâtre d'Aujourd'hui, 1994 (mai).

+ Les muses orphelines, mise en scène par René Richard Cyr, Montréal, Théâtre d'Aujourd'hui, 1994 (octobre).

+ Les muses orphelines, mise en scène par René Richard Cyr, Montréal, Théâtre d'Aujourd'hui, 1995.

+ Le voyage du couronnement, mise en scène par René Richard Cyr, Montréal, Théâtre du Nouveau Monde (TNM), 1995 (septembre).

+ Les muses orphelines, mise en scène par André Brassard, Montréal, Théâtre d'Aujourd'hui, 1998 (septembre).

\section{IV.3.5. Documents a diovisuels}

+ BOURDON, Luc [réal.], Yves FORTIN, Fernand HARVEY, Luce ROY, Colette LOUMÈDE, Synercom téléproductions, INRS - Culture et société, Télé-Québec, La culture dans tous ses états: le thêâtre, Montréal, Synercom téléproductions inc., 2000 (52 min 50 s).

+ COUlBOIS, Jean-Claude, Un miroir sur la scène, Montréal, Association coopérative de productions audio-visuelles, Office national du film du Canada, 1997, VHS, 107 min.

+ DESROSIERS, Robert, Michel Marc Bouchard: de toutes pièces, Prod. Radio-Québec, Montréal, Radio-Québec, VHS, s. d., 53 min.

+ DUCHESNE, Michel, Théâtre en jeu, Montréal, Cinéma 3180 B.E. inc. et Télé-Québec, 1997, VHS, 25 min 30 s, vidéocassette 1 de 5. 